\title{
Clinical Investigation on Gouqi Fushen Pills for Chronic Nephritis
}

\author{
Di ZOU ${ }^{1, a}$, Cheng-ji CUI ${ }^{2}$, Yin-ping WANG $^{3}$, Shou-lin ZHANG ${ }^{4, b,{ }^{*}}$ \\ ${ }^{1,2,3,4}$ Nephrology department, the Affiliated Hospital to Changchun University of \\ Chinese Medicine, Changchun, Jilin province, China. 130021. \\ a,aggiezou@163.com , b, shoulin-z@163.com \\ *Shou-lin ZHANG
}

Keywords: Gouqi fushen pills, Chronic nephritis, Clinical investigation

\begin{abstract}
Purpose:Observe the effectiveness and security of Gouqi fushen pills treating chronic nephritis. Method:Randomly divide 230 patients with chronic glomerular nephritis into two groups, treatment group and comparison group, each of 115 cases. Both groups orally take benazepril hydrochloride $10 \mathrm{mg}$ once a day, while treatment group orally takes a gouqi fushen pill three times a day, in a three-month period. Observe before and after treatment symptoms of both groups, as well as their $24 \mathrm{~h}$ quantitation of urine protein, counting of urine $\mathrm{RBC}$, and function changes of liver and kidney. Result: (1).5 drop-outs in treatment group, and 12 in comparison group, with the respective total effective rate of $83.64 \%$ and $47.57 \%$. The digital difference, according to statistics, has a statistical significance $(P<0.01)$. (2). Patients in treatment group have seen obvious alleviation of symptoms of osphyalgia, lack in strength and feverish palms and soles $(P<0.05)$, while comparison group haven't $(P>0.05)$. (3). Both groups have a distinct reduction in their $24 \mathrm{~h}$ quantitation of urine protein and counting of urine $\mathrm{RBC}$, with treatment group reduction higher than comparison group $(P<0.05)$. (4). Neither group has significant change in liver and kidney functions. Conclusion: Gouqi fushen pills are more effectively conducive than benazepril hydrochloride to cut down patients' $24 \mathrm{~h}$ quantitation of urine protein and counting of urine RBC. Also the pills can abate patients' suffer from osphyalgia, lack in strength and feverish palms and soles.
\end{abstract}

\section{Introduction}

Chronic glomerular nephritis, abbreviated as chronic nephritis, is a glomerular disease performing clinically as hematuresis,proteinuria,hydroncus and hypertension, with which about one third patients progress to ESRD after 10 years' paroxys ${ }^{[1]}$. Traditional Chinese medicine may have prominent efficacy for chronic nephritis, but as there is a lack of patent prescription, it mainly depends on decoction therapies. Gouqi fushen pills, developed by Dr. Zhen-chang Zhao, a prestigious professor of traditional Chinese medicine, have been eminent in efficacy since it first appeared to treat chronic nephritis over 20 years ago. To further verify the pills' effectiveness and security, we have set up this investigation.

\section{Materials and Methods}

\section{Case Source}

All 230 cases were chosen from both outpatients and inpatients of affiliated hospital of Changchun University of Chinese Medicine, dating from Oct. to Dec. 2014. The y were randomly divided two groups, each of 115 cases, out of which the treatment 
group had 60 male and 55 female patients aged from 16 to 65 , with comparison group having 56 male and 59 female patients aged from 19 to 68. To make it more explicit, the average age of both group was $42.8 \pm 9.2$ and $43.6 \pm 10.3$ respectively, with average disease course of $8.6 \pm 1.82$ and $7.8 \pm 1.69$ years, and a number of 45 versus 43 patients that had hypertension. Generally there were no remarkable difference in sexuality, age, disease course or patients' condition $(P>0.05)$.

\section{Diagnosing Criterion}

1) Western medicine diagnosis standard of chronic glomerular nephritis is according to $<$ Nephrology $>3$ rd edition [2]:urine abnormal RBC proportion over $70 \%$ in both continuous microscopic hematuria and/or gross hematuria. Normal renal function, with or without a small amount of $24 \mathrm{~h}$ proteinuria no more than $1.0 \mathrm{~g}$.

2) Belong to deficiency of spleen and kidney, with a damp heat and blood stasis. (According to the standards of TCM "Guiding Principles for New Traditional Medicine Clinical Investigation"[3].

3) Blood pressure controlled under $130 / 80 \mathrm{mmHg}$.

4) aged between 16 and 70 .

\section{Elimination Crite rion}

1) patients with hematuria or proteinuria caused by secondary glomerulopathy like purpura nephritis or lupus nephritis, urinary infections, acute post-infectious glomerular nephritis, hereditary renal diseases, syndrome of osphyalgia and hematuria, idiopathic hypercalciuria or athlete nephritis. 2) patients with severe organ diseases in heart, brain, liver or lung. 3) pregnant or lactating women, 4) lunatics.

\section{Grouping and Treatment Methods}

Randomly divide 230 patients with chronic glomerular nephritis into two groups, treatment group and comparison group, each of 115 cases. Both groups orally take benazepril hydrochloride $10 \mathrm{mg}$ once a day in a three-month period, provided by Beijing Novartis Pharma Ltd, while treatment group orally takes a gouqi fushen pill three times a day in a three-month period provided as Changchun healthcare medicine (96) No.1413 by manufacturing laboratory of affiliated hospital of Changchun University of Chinese Medicine.Patients should take anti-hypertensive calcium antagonist drugs if their blood pressure stays above $130 / 80 \mathrm{mmHg}$.

\section{Observing Index}

compare the before and after treatment figures in integral of syndrome, $24 \mathrm{~h}$ quantitation of urine protein, counting of urine $\mathrm{RBC}$, and function changes of liver and kidney.

\section{Efficacy Judging Method}

Efficacy Judging Criterion. Clinical control,excellence, efficiency and futility, according to"Guiding Principles for New Traditional Medicine Clinical Investigation" [3].Use gradational scoring method to observe before and after treatment changes in traditional medicine syndrome, as following details in table 1 . 
Table 1 Gradational Scoring Criterion for Syndrome

\begin{tabular}{|c|c|c|c|}
\hline item & Lumbar spine pain & fatigue & feverish palms and soles \\
\hline 0 points & none & none & none \\
\hline 2 points & $\begin{array}{c}\text { Occasional or after } \\
\text { exhaustion }\end{array}$ & $\begin{array}{c}\text { Occasional, slight, Not resistant to } \\
\text { labor }\end{array}$ & At times \\
\hline 4 points & $\begin{array}{c}\text { A dull pain relief } \\
\text { after rest }\end{array}$ & Manual labor insisting & $\begin{array}{c}\text { Need to expose hands and } \\
\text { feet }\end{array}$ \\
\hline 6 points & $\begin{array}{l}\text { Obvious pain, } \\
\text { Can't stand or } \\
\text { walk long,action } \\
\text { affected }\end{array}$ & $\begin{array}{c}\text { Fatigue after normal actions, even } \\
\text { normal daily life Consistent } \\
\text { fatigue,Can't complete daily } \\
\text { activity }\end{array}$ & Want to touch Cool things \\
\hline
\end{tabular}

Evaluation Criterion for Syndrome Efficacy. Adopt Nimodiping method to calculate integral changes [3].

\section{Statistical Method}

Analyze figures with statistical software of SPSS 17.0, illustrating calculated data with $\left(\bar{x}_{ \pm s}\right)$, and counting data with chi-square test, in-group comparison with paired $\mathrm{T}$ test, and last but not least, inter-group comparison with independent sample T test, in which $P<0.05$ means a difference with statistical significance.

\section{Results}

\section{Overall Efficacy Comparison}

The total effective rate of treatment group and comparison group is respectively $83.64 \%$ and $47.57 \%$. The digital difference, according to statistics, has a statistical significance $(P<0.01)$,as shows in table2.

Table 2. Overall efficiency figures in both groups(cases)

\begin{tabular}{ccccccc}
\hline group & $\mathrm{n}$ & Clin ical control excellence & efficiency & futility & Overall efficiency $(\%)$ \\
\hline treatment & 110 & 6 & 28 & 58 & 18 & $83.64^{*}$ \\
comparison & 103 & 2 & 6 & 41 & 54 & 47.57 \\
\hline
\end{tabular}

Note: compare with comparis on group, $P<0.01$

\section{Comparison of Gradational Scoring for TCM Syndrome}

Comparing with before treatment and after treatment,TCM Syndrome of treatment group have remarkably improved $(P<0.05)$, TCM Syndrome of comparison group nearly didn't change $(P>0.05)$. Compared between two group, TCM Syndrome of treatment group is better than comparison group $(P<0.05)$. Details follows in table3. 
Table3:before and after treatment, Compare of Gradational Scoring for TCM Syndrome

\begin{tabular}{cccccc}
\hline Group & $\mathrm{n}$ & & $\begin{array}{c}\text { Lumbar spine } \\
\text { pain }\end{array}$ & fatigue & $\begin{array}{c}\text { feverish palms } \\
\text { and soles }\end{array}$ \\
\hline \multirow{2}{*}{ treatment group } & 110 & before & $2.50 \pm 0.94$ & $2.70 \pm 1.02$ & $1.76 \pm 0.49$ \\
& & after & $1.51 \pm 0.47^{\star \Delta}$ & $1.79 \pm 0.82^{\star \Delta}$ & $1.59 \pm 0.47^{\star \Delta}$ \\
comparison group & 103 & before & $2.47 \pm 0.95$ & $2.67 \pm 1.14$ & $1.83 \pm 0.46$ \\
& & after & $2.38 \pm 0.85$ & $2.65 \pm 1.13$ & $1.79 \pm 0.43$ \\
\hline
\end{tabular}

Note: compare between treatment group before and after treatment, $P<0.05$; compare with comparison group, ${ }^{\Delta} P<0.05$

\section{Pre-post Treatment Figures of Counting of Urine RBC and 24h Quantitation of Urine Protein}

Compared with pre-treatment and after treatment, both figures in treatment group have remarkably decreased, which has statistical significance $(P<0.05)$. After treatment, 24h quantitation of urine protein of comparison group decreased $(P<0.05)$, urine RBC of comparison group nearly didn't change $(P>0.05)$.Compared between two group, the curative effect of treatment group is better than comparison group $(P<0.05)$. Details follows in table4.

Table4:before and after treatment counting of urine RBC and 24h UTP for two groups

\begin{tabular}{cccc}
\hline group & & RBC & 24h UTP \\
\hline \multirow{2}{*}{ treatment group } & before & $26.46 \pm 6.30$ & $0.96 \pm 0.22$ \\
& after & $9.20 \pm 1.56^{\star \Delta}$ & $0.25 \pm 0.08^{\star \Delta}$ \\
Comparison group & before & $23.24 \pm 3.72$ & $0.85 \pm 0.18$ \\
& after & $21.17 \pm 3.32^{*}$ & $0.48 \pm 0.13^{*}$ \\
\hline
\end{tabular}

Note: compare between treatment group before and after treatment, ${ }^{*} P<0.05$; compare with comparis on group, ${ }^{\Delta} P<0.05$

\section{Comparison of Security}

Neither group witnessed significant adverse medicinal reactions, all patients having the no change of liver and renal functions before and after treatment.

\section{Discussion}

As the main clinical performances of chronic glomerular nephritis, hematuresis and proteinuria also act as important hazardous factors during renal disease progressive development[4]. When talking about treatment for chronic nephritis, modern medicine still mainly depends on RAAS system blockers, hormones or cellular poisons, which primarily aims at patients with $24 \mathrm{~h}$ quantitation of urine protein over $1.0 \mathrm{~g}$. But for those under $1.0 \mathrm{~g}$, or with simple hematuresis, there has been a lack of effective means of treatment, meaning that patients of this kind hasn't been taken seriously before. But recent studies have shown that ${ }^{[5]}$ pathological injuries of these patients may not be mild and their conditions may progress at any time.What's more, Dr. Weibo 
Le[6]pointed out that only with a controlled proteinuria under $0.5 \mathrm{~g} / \mathrm{d}$ can the risk of outcome measures happening be significantly reduced. He also made it clear that microscopic hematuria is related to long-term prognosis, with a 2 times risk of entering outcome measures as each 10 times RBC counts. Accordingly, patients with simple hematuresis or light proteinuria should be given active treatment to prevent pathological deteriorating to ESRD.

As belongs to the ambit of hematuresis, hydroncus and osphyalgia,chronic nephritis is often thought to result from pyretic toxicity harming renal channels due to a damp heat in the bladder. Medical scientists propose a therapy based on cooling for diuresis and hemostasis, but have recurrent attacks after slight efficacy without radical cure. Dr.Zhen-chang Zhao holds a different opinion that the primary cause of chronic nephritis comes from the deficiency of both the spleen and kidney as well as a wet and blood stasis.As kidney functions body fluids while spleen transmits and distributes the fluids, the asthenia of the both may form an interior dampness, which is hard to eradicate with the traditional way of cooling for diuresis and hemostasis. Consequently, to fully heal chronic nephritis and consolidate body, doctors must lay stress on kidney invigorating and spleen strengthening. Based on this theory and his clinical experience for many years, Dr. Zhao has developed the gouqi fushen pills, which has been in application as interior medicine for about 20 years since 1996. The pills are mainly utended to cure hematuresis and proteinuria of chronic nephritis, getting considerable economic benefit.Chinese wolfberry, mild and sweet, is quite useful to reinforce masculine power and to nourish blood, while milkvetch, spleen tonifying and middle warmer strengthening, can reinforce gas and blood and discharge turbidity, both of which contribute to the main prescription of the pills. At the same time, there goes some auxiliary ingredients, such as eclipta, ligustri lucidi and Radix Rehmanniae that also enrich the blood, cogongrass rhizome that clears heat and stanches bleeding, Cirsium japonicum, Cirsium setosum, and Sophorae that also cools the blood.Dr. Zhao believes that pathogen usually intrudes into collateral in protracted disease, and that there will be blood stasis in chronic illness. So he added radix paeoniae, angelica, carthamus tinctorious and heterophyllus to remove blood stasis for a better renal microcirculation, with cortex moutan, cape jasmine and rheum officinale to relax veins, and also some poria cocos to eliminate pathogen. All these make up of the pills, a method of both reinforcement and reduction, contributing to boost the essence of human body.

\section{Conclusion}

Results shows that the overall efficiency of treatment is impressively higher than comparison group, respectively $83.64 \%$ and $47.57 \%(P<0.01)$. Also does it have a remarkable result in ameliorating patients' clinical symptoms and in reducing urine RBC and proteinuria $(P<0.01)$. With this outcome, not only can gouqi fushen pills efficiently decrease patients' urine $\mathrm{RBC}$ and $24 \mathrm{~h}$ proteinuria, the pills can also relief them from clinical symptoms like lumbar spine pain,fatigue and feverish palms and soles.Moreover, the higher efficacy than ACEI class effect only together with the harmlessness to liver and renal functions have proved it worthy to further research and development.

\section{Ackno wledge ment}

This research was financially supported by project as follows: Jilin Provincial Science \& Technology Department (20130206071YY); Jilin Province Education Department 
"Twelfth Five Year Plan" science and technology research project (No. 65 of 2014); Jilin Province Administration of traditional Chinese medicine science and technology project (No. 2014-ZC47)

\section{Reference}

[1]Zhang Hong,KDIGO guide interpretation:Treatment of IgA nephritis (in Chinese). Chinese Journal of Practical Internal Medicine. 2012,32(12):925-927.

[2]Wang Hai-yan,Nephrology 3rd edition(in Chinese).Beijing People's Medical Publishing House, 2008,937.

[3]Zheng Xiaoyu,Guiding Principles for New Traditional Medicine Clinical Investigation (in Chinese).Beijing China Medical Science and Technology Publishing House, 2002. 156-162.

[4]Takaya K,Koya D,Isono M,et al.Involvement of ERK pathway in albumin induced MCP-1 expression in mouse proximal tu-bular cells. Am $\mathrm{J}$ Physiol Renal Physiol,2003,284( 5) :F1037-F1045.

[5]Lv Ji-cheng, Wang Hai-yan, Pathological analysis of IgAN with simple hematuria and (or) mild proteinuria(in Chinese).Chinese Journal of Renal Disease, 2004,12(6): 418-420.

[6]Le Wei-bo, Liang Shao-shan, Deng Kang-ping, etc. Long-term Prognosis and Risk Factors Analysis for 1126 Chinese Han dynasty adult Patients with Ig AN(in Chinese). Journal of kidney disease and dialysis kidney transplantation,2011,20(2):101-108. 